\title{
Unilateral vestibular schwannoma in a patient with schwannomatosis in the absence of LZTR1 mutation
}

\author{
Gautam U. Mehta, MD, ${ }^{1,2}$ Michael J. Feldman, BA, ${ }^{1}$ Herui Wang, PhD, ${ }^{1}$ Dale Ding, MD, ${ }^{2}$ and \\ Prashant Chittiboina, MD ${ }^{1}$ \\ ${ }^{1}$ Surgical Neurology Branch, National Institute of Neurological Disorders and Stroke, National Institutes of Health, Bethesda, \\ Maryland; and ${ }^{2}$ Department of Neurosurgery, University of Virginia Health System, Charlottesville, Virginia
}

\begin{abstract}
The presence of vestibular schwannomas has long been considered an exclusion criterion for the diagnosis of schwannomatosis. Recently, 2 cases of vestibular schwannoma were reported in patients with schwannomatosis, leading to a revision of the diagnostic criteria for this genetic disorder. Overall, the relative infrequency of vestibular schwannomas in schwannomatosis is unexplained, and the genetics of this uncommon phenomenon have not been described. The authors report on a family with clinical manifestations consistent with schwannomatosis, including 4 affected members, that was identified as having an affected member harboring a unilateral cerebellopontine angle mass with extension into the internal auditory canal. Radiologically, this mass was consistent with a vestibular schwannoma and resulted in a symptomatic change in ipsilateral hearing (word recognition $86 \%$ at $52 \mathrm{~dB}$ ) and increased latency of the wave I-V interval on auditory brainstem response testing. The patient was found to be negative for a germline mutation of NF2 and LZTR1, and her affected mother was found to harbor neither NF2 nor SMARCB1 mutations on genetic testing. Although vestibular schwannomas have been classically considered to not occur in the setting of schwannomatosis, this patient with schwannomatosis and a vestibular schwannoma further confirms that schwannomas can occur on the vestibular nerve in this syndrome. Further, this is the first such case found to be negative for a mutation on the LZTR1 gene.

http://thejns.org/doi/abs/10.3171/2015.11.JNS151766
\end{abstract}

KEY WORDS cranial nerve; LZTR1; schwannomatosis; vestibular schwannoma

$\mathrm{S}$ CHWANNOMATOSIS is a rare tumor predisposition syndrome, the prevalence of which has yet to be precisely defined. ${ }^{4}$ The syndrome is characterized by the development of multiple intracranial and spinal schwannomas, and can be either familial (15\%-25\% of cases) or sporadic. ${ }^{6}$ Although schwannomatosis is grouped among the neurofibromatoses, it results in histologically uniform tumors and is believed to occur substantially less frequently in the general population than neurofibromatosis Type 1 ( 1 in 3000 individuals) or Type 2 ( 1 in 33,000 to 40,000 individuals). ${ }^{4}$

The diagnostic criteria for schwannomatosis has, for many years, excluded patients harboring a vestibular schwannoma. ${ }^{2,3}$ Recently, 2 cases of schwannomatosis with unilateral vestibular schwannomas were identified. ${ }^{9}$ Based on the report of these 2 cases from a single institution, a recent revision of the diagnostic criteria was proposed to include patients with unilateral, but not bilateral, vestibular schwannomas. ${ }^{6}$ Since this initial report, no further cases of vestibular schwannoma in patients with schwannomatosis have been reported. The genetic mechanisms of schwannomatosis are still being elucidated; however, subsets of patients have been found to have mutations in SMARCBI (also known as INII) and LZTRII.,5 A mutation in the LZTRI gene was identified in one of the 2 previously reported patients with unilateral vestibular schwannoma and has been suggested to possibly confer an increased risk of vestibular schwannoma in the setting of schwannomatosis. ${ }^{8}$

Herein, we present a patient with schwannomatosis who harbored a unilateral vestibular schwannoma. This is the third such case reported to date and lends further support to the change in the diagnostic criteria for schwannomatosis to include patients with unilateral vestibular schwannomas. Furthermore, we did not identify an exomic mutation in the LZTRI gene in this patient, suggesting that this disease phenotype is not restricted to a specific genetic mutation. 


\section{Case Report}

A 51-year-old woman presented to our neurofibromatosis Type 2 (NF2) clinic with a right-sided trigeminal schwannoma, a right-sided cerebellopontine angle tumor radiologically consistent with a vestibular schwannoma, and a punctate lumbar schwannoma (Fig. 1). She had originally been diagnosed with NF2 at 50 years of age and subsequently underwent stereotactic radiosurgery $(20 \mathrm{~Gy}$ margin dose) for the trigeminal schwannoma. The patient noted several years of persistent difficulty with taste on the anterior portion of her tongue and subtle right-sided hearing changes that made it difficult to play the violin. On neurological examination, she had diminished sensation on the right side of her face in maxillary (V2) and mandibular (V3) distributions, with normal facial motor function. On audiological testing of her right ear, she achieved word recognition of $76 \%$ at $57 \mathrm{~dB}$ and $80 \%$ at $85 \mathrm{~dB}$, with normal left-sided hearing function. Auditory brainstem response testing revealed increased latency of the wave $\mathrm{I}-\mathrm{V}$ interval on the right.

\section{Family and Genetic Testing History}

The patient's maternal grandfather had a benign spinal tumor resected in his $60 \mathrm{~s}$, her mother had a spinal schwannoma resected at the age of 72 years, and her sister had been found on neuroimaging to have multiple lumbar schwannomas (Fig. 2). The patient's mother had previous genetic testing that was negative for both $N F 2$ and SMARCBl mutations. The patient herself had prior genetic testing that was negative for an NF2 mutation. Since the cerebellopontine angle lesion was presumed to be a vestibular schwannoma, a diagnosis of NF2 had initially been considered, with possible mosaicism, explaining the negative genetic test result for the NF2 mutation.

We performed Sanger sequencing using polymerase chain reaction (PCR) amplification and TA Cloning to evaluate all coding and junctional regions of the LZTRI gene in blood-extracted DNA. No missense mutations were observed, confirming that the patient did not harbor a germline LZTRI mutation.

\section{Management}

Based on her lesions, genetics, and family history, a

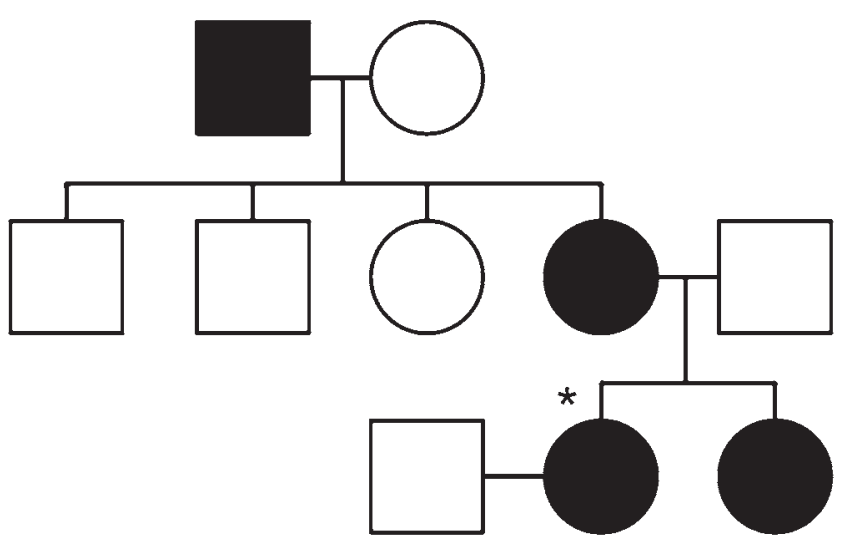

FIG. 2. Family pedigree, with affected patients (black) including the current patient (asterisk), sister (multiple spinal schwannomas), mother (resected spinal schwannoma), and maternal grandfather (benign spinal tumor). Circles denote females; squares, males.

diagnosis of familial schwannomatosis was made. The patient was followed for 5 years with serial neuroimaging and audiological testing. Over this time period, the trigeminal schwannoma that had been treated with radiosurgery continued to diminish in size. Interestingly, the vestibular schwannoma, which was not in the radiation field employed to treat the trigeminal lesion, reduced in size as well during the follow-up period (Fig. 1). Finally, the lumbar schwannoma grew minimally during the follow-up period and remained asymptomatic. Based on these findings, continued observation alone was recommended.

\section{Discussion}

Schwannomatosis is a rare tumor predisposition syndrome that is considered to be a form of neurofibromatosis, in which patients present with multiple intracranial and spinal schwannomas. Approximately $15 \%-25 \%$ of cases are familial, while the majority are sporadic. ${ }^{6}$ Proposed updated clinical diagnostic criteria include 1) having 2 or more non-intradermal schwannomas with pathological confirmation in the absence of bilateral vestibular schwannomas, and 2) having 1 pathologically confirmed non-intradermal schwannoma and an affected first-de-
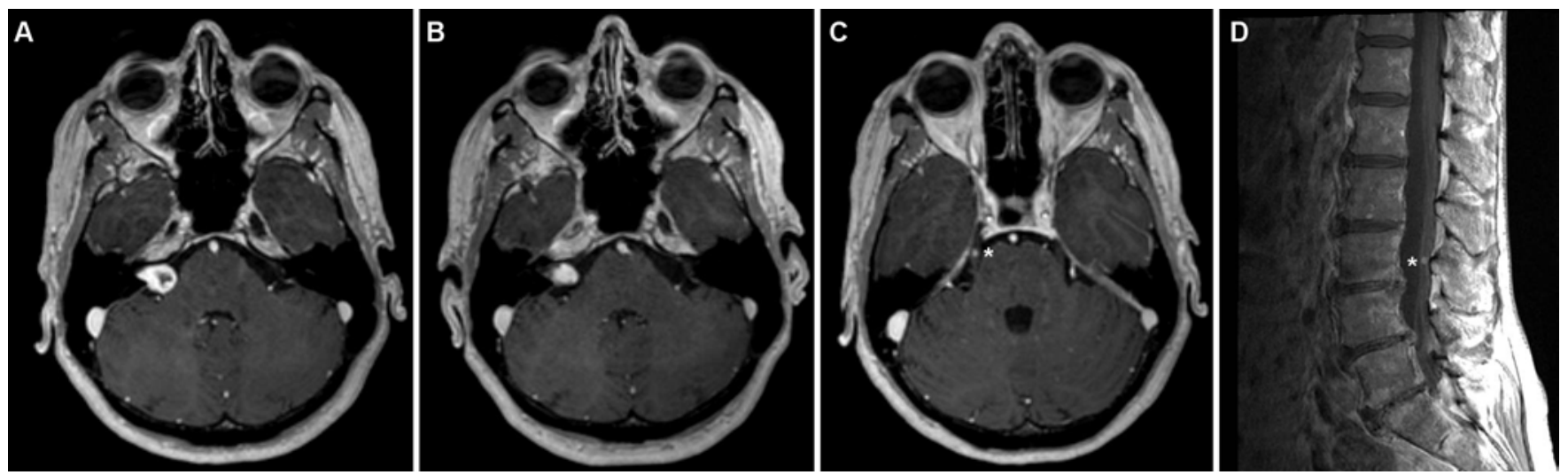

FIG. 1. Axial T1-weighted MR images showing a right-sided vestibular schwannoma (A) that diminished in size at the end of a 5 -year radiographic follow-up period (B), a right-sided trigeminal schwannoma (asterisk, C), and a lumbar schwannoma on the sagittal image (asterisk, D). 
gree relative. ${ }^{6}$ Patients with 2 or more non-intradermal schwannomas without pathological confirmation may be considered for inclusion as well. For many years, the presence of a vestibular schwannoma was considered an exclusion criterion for the diagnosis of schwannomatosis. Revised criteria have since been proposed, based solely on the report of 2 patients with unilateral vestibular schwannoma out of $178(1.1 \%)$ with schwannomatosis in the Manchester Schwannomatosis database. ${ }^{9}$

\section{Genetics}

While a germline mutation in the NF2 gene is considered exclusionary for schwannomatosis, germline mutations in SMARCBI (also known as INII) and LZTRI have been identified in patients with schwannomatosis. ${ }^{1,5,7} \mathrm{~A}$ recent analysis of the Manchester Schwannomatosis database demonstrated that $37.5 \%$ and $22 \%$ of patients with familial and sporadic schwannomatosis, respectively, harbored germline mutations in LZTR $1 .{ }^{8}$ This included 1 patient with a unilateral vestibular schwannoma who was previously described, leading the authors to postulate that this specific phenotype might be mutation specific. ${ }^{9}$

We describe the third reported case of a patient with schwannomatosis harboring a vestibular schwannoma. This is the first reported case outside of the Manchester Schwannomatosis database. Furthermore, this patient is unique in that she does not harbor germline mutations in either the SMARCBI or LZTRI genes.

\section{Implications for Management}

Given our patient's initially incorrect diagnosis of NF2, this case serves as an important example that patients harboring a vestibular schwannoma and additional nonvestibular schwannomas may be misdiagnosed, potentially resulting in a general underdiagnosis of schwannomatosis. In fact, Smith et al. identified 39 patients with a unilateral vestibular schwannoma and at least 1 additional schwannoma, of whom $2(5 \%)$ harbored a germline mutation in the LZTRI gene. ${ }^{8}$ Awareness of this increased risk of tumor development and familial transmission is critical to the management and counseling of patients with this presentation. Furthermore, a thorough family history should be considered requisite for such patients, and genetic testing may be of value to patients who are considering having children. Finally, as such tumor predisposition syndromes are often characterized by new lesion development over time, the neurosurgical management paradigm for affected patients should shift from one achieving curative resection to one of surveillance with intervention for symptomatic lesions.

\section{Conclusions}

This case strengthens the recommendation that patients with a unilateral vestibular schwannoma might be considered for the diagnosis of familial schwannomatosis. Furthermore, it is the first to demonstrate that this disease phenotype is not restricted to patients with mutations in the LZTRI gene.

\section{Acknowledgments}

This research was supported by the Intramural Research Program of the National Institute of Neurological Disorders and Stroke at the National Institutes of Health (NIH).

\section{References}

1. Boyd C, Smith MJ, Kluwe L, Balogh A, Maccollin M, Plotkin SR: Alterations in the SMARCB1 (INI1) tumor suppressor gene in familial schwannomatosis. Clin Genet 74:358-366, 2008

2. Evans DG, Huson SM, Donnai D, Neary W, Blair V, Newton V, et al: A clinical study of type 2 neurofibromatosis. Q J Med 84:603-618, 1992

3. MacCollin M, Chiocca EA, Evans DG, Friedman JM, Horvitz R, Jaramillo D, et al: Diagnostic criteria for schwannomatosis. Neurology 64:1838-1845, 2005

4. Melean G, Sestini R, Ammannati F, Papi L: Genetic insights into familial tumors of the nervous system. Am J Med Genet C Semin Med Genet 129C:74-84, 2004

5. Piotrowski A, Xie J, Liu YF, Poplawski AB, Gomes AR, Madanecki P, et al: Germline loss-of-function mutations in LZTR1 predispose to an inherited disorder of multiple schwannomas. Nat Genet 46:182-187, 2014

6. Plotkin SR, Blakeley JO, Evans DG, Hanemann CO, Hulsebos TJM, Hunter-Schaedle K, et al: Update from the 2011 International Schwannomatosis Workshop: From genetics to diagnostic criteria. Am J Med Genet A 161A:405416,2013

7. Rousseau G, Noguchi T, Bourdon V, Sobol H, Olschwang S: SMARCB1/INI1 germline mutations contribute to $10 \%$ of sporadic schwannomatosis. BMC Neurol 11:9, 2011

8. Smith MJ, Isidor B, Beetz C, Williams SG, Bhaskar SS, Richer W, et al: Mutations in LZTR1 add to the complex heterogeneity of schwannomatosis. Neurology 84:141-147, 2015

9. Smith MJ, Kulkarni A, Rustad C, Bowers NL, Wallace AJ, Holder SE, et al: Vestibular schwannomas occur in schwannomatosis and should not be considered an exclusion criterion for clinical diagnosis. Am J Med Genet A 158A:215219,2012

\section{Disclosures}

The authors report no conflict of interest concerning the materials or methods used in this study or the findings specified in this paper.

\section{Author Contributions \\ Conception and design: Mehta, Ding, Chittiboina. Acquisition of data: Mehta, Feldman, Wang. Analysis and interpretation of data: Mehta, Feldman, Wang. Drafting the article: Mehta, Feldman. Critically revising the article: all authors. Reviewed submitted version of manuscript: all authors. Approved the final version of the manuscript on behalf of all authors: Mehta. Study supervi- sion: Mehta.}

\section{Correspondence}

Gautam U. Mehta, Surgical Neurology Branch, National Institute of Neurological Disorders and Stroke, 10 Center Dr., Rm. 3D20, Bethesda, MD 20892. email: gautam.mehta@nih.gov. 